\title{
Heterosynaptic Modulation of Motor Cortical Plasticity in Human
}

\author{
Zhen Ni, Carolyn Gunraj, Paramjit Kailey, Robin F. H. Cash, and Robert Chen \\ Division of Neurology, Krembil Neuroscience Centre and Toronto Western Research Institute, University Health Network, University of Toronto, Toronto, \\ Ontario, M5T 2S8 Canada
}

\begin{abstract}
Inductions of long-term potentiation (LTP) and depression (LTD) are modulated if they are preceded by a priming protocol, in a manner consistent with metaplasticity. Depotentiation refers to reversal of LTP by a subsequent protocol that has no effect by itself. Paired associative stimulation (PAS) at interstimulus interval of $25 \mathrm{~ms}$ (PAS25) and $10 \mathrm{~ms}$ (PAS10) produces spike timing-dependent LTP-like and LTD-like effects in human primary motor cortex. Continuous theta burst stimulation (cTBS) with 600 pulses produces an LTD-like effect, whereas cTBS with 150 pulses (cTBS150) has no effect by itself. We investigated whether cortical plasticity induced by PAS can be modulated by heterosynaptic inputs of cTBS150. PAS25 and PAS10 primed and followed by cTBS150 were compared withPAS25 and PAS10 alone. Motor evoked potential (MEP) amplitude, recruitment curve, and intracortical circuits including short-interval intracortical inhibition (SICI), long-interval intracortical inhibition (LICI), intracortical facilitation, and short-latency afferent inhibition were measured before and after the interventions. After PAS25 alone, MEP amplitude increased while intracortical circuits did not change. A priming cTBS150 enhanced the effects of PAS25 with further increase in MEP amplitude and led to reduction in SICI and LICI. PAS25 followed by cTBS150 led to reduced MEP amplitude and increased LICI and SICI. Both priming and following cTBS150 reversed the LTD-like effect produced by PAS10 with little change in intracortical circuits. We conclude that cortical plasticity induced by PAS and cTBS interacts in a heterosynaptic and bidirectional manner. The order of the interventions determines whether the underlying mechanisms are related to metaplasticity or depotentiation.
\end{abstract}

Key words: cortical plasticity; depotentiation; metaplasticity; motor evoked potential; transcranial magnetic stimulation

\section{Introduction}

Each neuron receives thousands of synapses from thousands of neurons. Activation of these synapses under certain conditions induces synaptic plasticity. The motor cortex plays a primary role in motor control and the occurrence of transient or lasting plasticity in human motor cortex has been documented (Sanes and Donoghue, 2000). The best known neuronal mechanisms underlying cortical plasticity are long-term potentiation (LTP) and long-term depression (LTD; Malenka and Bear, 2004). Repetitive transcranial magnetic stimulation (TMS) offers a unique opportunity to investigate the LTP and LTD-like effects in human brain at the systems level (Hallett, 2007). Continuous theta burst stimulation (cTBS) has been introduced as a powerful protocol to produce LTD-like effects in the motor cortex while TBS delivered in an intermittent mode produces LTP-like effects (Huang et al., 2005). Paired associative stimulation (PAS) involves pairing sensory afferent inputs with direct activation of motor cortex by

Received Nov. 6, 2013; revised March 28, 2014; accepted April 21, 2014.

Author contributions: Z.N. and R.C. designed research; Z.N., C.G., and P.K. performed research; Z.N. and R.C. analyzed data; Z.N., R.F.H.C., and R.C. wrote the paper.

This study was supported by the Canadian Institutes of Health Research (MOP 62917).

The authors declare no competing financial interests.

Correspondence should be addressed to Dr. Robert Chen, 7MC-411, Toronto Western Hospital, 399 Bathurst

Street, Toronto, Ontario, M5T 2S8, Canada. E-mail: robert.chen@uhn.ca.

DOI:10.1523/JNEUROSCI.4714-13.2014

Copyright $\odot 2014$ the authors $\quad 0270-6474 / 14 / 347314-08 \$ 15.00 / 0$
TMS at specific timings. If the median nerve stimulation is delivered $\sim 25 \mathrm{~ms}$ before TMS (PAS25), the two stimuli arrive at the motor cortex at about same time and LTP-like effect is generated. If the stimulus interval is $\sim 10 \mathrm{~ms}$ (PAS10), TMS pulse is applied before median nerve stimulation reaches the cortex and LTD-like effect is generated. Spike timing-dependent plasticity is considered the most likely mechanism underlying the effects of PAS (Stefan et al., 2000; Wolters et al., 2003).

Cortical plasticity is modulated by several different synaptic mechanisms, depending on the activation history of the involved synapses. The induction of LTP is facilitated if a LTP protocol is preceded by a LTD protocol, a mechanism termed metaplasticity (Bienenstock et al., 1982). Depotentiation refers to reversal of LTP by which LTP is abolished by a following protocol that has no effect by itself (Huang et al., 1999; Kulla and ManahanVaughan, 2000). Homosynaptic plasticity refers to alterations in synaptic efficacy in the same synapses that receive plasticityinducing input. The modulation of homosynaptic LTP-like effect with metaplasticity (Hamada et al., 2008) and depotentiation (Huang et al., 2010) has been reported in human motor cortex. Induction of heterosynaptic plasticity, which involves interactions among different synapses where inputs to one type of synapse lead to long-range cross-compartment changes in remote synapses, has been demonstrated in rodent cortical slices (Abraham, 2008; Turrigiano, 2008; Chistiakova and Volgushev, 2009). Studies at the systems level in human reported the modulation of 
cortical plasticity by a priming protocol with heterosynaptic inputs (Siebner et al., 2004; Nitsche et al., 2007; Jung and Ziemann, 2009). However, bidirectional changes involving different underlying mechanisms determined by the order of two plasticity protocols have not been tested. To address this, we investigated whether PAS-induced effects can be modulated by a short train of cTBS with 150 pulses (cTBS150), which does not change cortical excitability by itself. We hypothesize that the priming protocol of cTBS150 will enhance the LTP-like effects produced by PAS25 and reverse the LTD-like effects produced by PAS10 due to metaplasticity. On the other hand, cTBS150 following PAS25 will abolish the LTP-like effects produced by PAS25 with depotentiation.

\section{Materials and Methods}

Subjects. We studied 14 right-handed (Oldfield, 1971) healthy subjects (6 females and 8 males, aged $35.1 \pm 3.4$ years). All subjects provided written informed consent in accordance with the Declaration of Helsinki. The protocol was approved by the University Health Network (Toronto) Research Ethics Board.

Electromyographic recording. Surface electromyograms were recorded from right abductor pollicis brevis (APB), first dorsal interosseous (FDI), and abductor digiti minimi (ADM) muscles with $9 \mathrm{~mm}$ diameter $\mathrm{Ag}$ $\mathrm{AgCl}$ surface electrodes. The active electrode was placed over the muscle belly, and the reference electrode over the metacarpophalangeal joint of the finger. The signal was amplified $(1000 \times)$, bandpass filtered $(20 \mathrm{~Hz}-$ $2.5 \mathrm{kHz}$; Intronix Technologies Model 2024F), digitized at $5 \mathrm{kHz}$ by an analog-to-digital interface (Micro1401; Cambridge Electronics Design, Cambridge, UK) and stored in a computer for off-line analysis.

PAS. PAS25 was delivered pairing median nerve stimulation followed by a TMS pulse at an interstimulus interval of $25 \mathrm{~ms}$. PAS 10 was delivered at an interstimulus interval of $10 \mathrm{~ms}$. One hundred and eighty pairs of stimulations were delivered in a frequency of $0.1 \mathrm{~Hz}(30 \mathrm{~min})$ (Stefan et al., 2000; Wolters et al., 2003). Median nerve stimulation was applied at the right wrist by a Digitimer DS7A constant current stimulator (pulse width $0.2 \mathrm{~ms}$ ) with standard bar electrodes. The cathode was positioned proximally. The stimulus intensity was three times sensory threshold. The sensory threshold was the lowest stimulus intensity felt by the subject. The TMS was applied to the left primary motor cortex with a figureof-eight-shaped coil (outside diameter of each loop was $9.5 \mathrm{~cm}$ ) connected to a Magstim $200^{2}$ stimulator. The handle of the coil pointed backward at $30-45^{\circ}$ from the mid-sagittal line, approximately perpendicular to the central sulcus. Monophasic pulses, which produced posterior-anteriorly directed current in the brain were used to deliver TMS. TMS with this current direction activates pyramidal neurons transsynaptically and predominantly generates early indirect waves in the spinal cord (Kaneko et al., 1996; Di Lazzaro et al., 2001). The APB muscle was selected as the target muscle as it is innervated by median nerve. The optimal position for activation of the right APB muscle was marked with a pen as the motor hot spot. TMS intensity was set at " $1 \mathrm{mV}$ " for each subject, which was defined as the lowest TMS intensity needed to generate motor evoked potentials (MEPs) of $>1 \mathrm{mV}$ in at least 5 of 10 trials in the target APB muscle when the muscle was completely relaxed. We also monitored the MEP in the adjacent nontarget FDI and ADM muscles to compare the PAS-induced changes in the target and adjacent muscles.

cTBS. cTBS150 consisted of 50 bursts containing three TMS pulses of $50 \mathrm{~Hz}$ repeated at $200 \mathrm{~ms}(5 \mathrm{~Hz})$ for a duration of $10 \mathrm{~s}$. This is a quarter of the number of pulses used in a standard cTBS protocol (Huang et al., 2005), and previous studies showed that it does not produce cortical plastic changes by itself (Huang et al., 2010, 2011). cTBS150 was delivered with biphasic pulses generated by a Magstim Super Rapid ${ }^{2}$ stimulator. Biphasic pulse produces both posterior-anteriorly and anterior-posteriorly directed currents in the brain at different phases. Therefore, the neuronal populations activated by the biphasic pulses are not identical to those activated by the monophasic pulses. cTBS150 used the stimulus intensity of $80 \%$ active motor threshold. Active motor threshold was the minimum intensity needed to evoke MEPs of $>100 \mu \mathrm{V}$ in at least 5 of 10

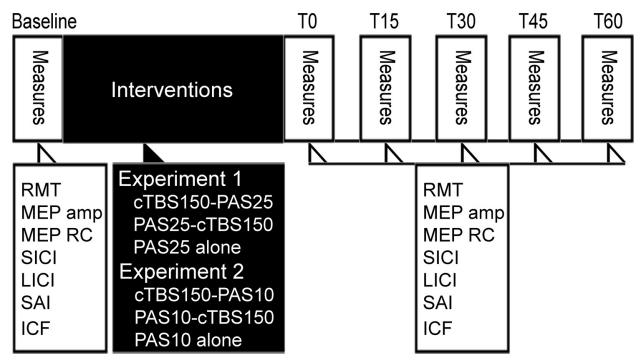

Figure 1. Experimental design. Two experiments were performed. Three different interventional protocols were compared in each experiment. The interventional protocols were PAS25 primed by cTBS150 (cTBS150-PAS25), PAS25 followed by cTBS150 (PAS25-CTBS150), and PAS25 alone in Experiment 1. For Experiment 2, the interventional protocols were CTBS150PAS10, PAS10-CTBS150, and PAS10 alone. Measurements were taken before (baseline), immediately after, and up to $60 \mathrm{~min}$ after each interventional protocol. The measurements were RMT, MEP amplitude, MEP recruitment curve, SICI, LICI, SAI, and ICF. CTBS150, CTBS with 150 pulses; MEP RC, MEP recruitment curve; PAS25 (or PAS10), PAS with 25 ms (or $10 \mathrm{~ms}$ ) interstimulus interval between median nerve stimulation and TMS.

trials when the subject maintained $20 \%$ maximum contraction of APB muscle aided by audiovisual feedback of electromyographic activity. Active motor threshold was determined with the Super Rapid ${ }^{2}$ stimulator.

Experimental design. We performed two experiments to investigate whether cTBS150 modulated the LTP-like effects produced by PAS25 (Experiment 1) and LTD-like effects produced by PAS10 (Experiment 2). Eleven subjects participated in Experiment 1 and 10 subjects participated in Experiment 2 ( 7 subjects participated for both experiments). Three interventional protocols cTBS150-PAS25 (or PAS10), PAS25 (or PAS10)-cTBS150, and PAS25 (or PAS10) alone were compared in each experiment (Fig. 1). For Experiment 1, the cTBS150-PAS25 protocol tested the effects of priming cTBS150 on PAS25. The PAS25-cTBS150 protocol tested whether PAS25-induced LTP-like effect was canceled by a subsequent CTBS150. The modulation of PAS10 by priming and following cTBS150 was tested in a similar way in Experiment 2. Each intervention was performed on a separate day at least 1 week apart in a random order. Measurements were taken before (baseline) and immediately, 15, 30, 45, and $60 \mathrm{~min}$ (T0-T60) after each interventional protocol.

We performed two control experiments to confirm the results of the main experiments. Ten subjects were tested (all participated in main Experiment 1) in the first control experiment to verify that cTBS150 alone did not produce any measurable effect by itself. The experimental procedure was the same as the main experiment. Seven subjects (all participated in the main experiments) were tested in the second control experiment to address whether the modulation of PAS-induced effects by cTBS150 was specific to the target (APB) muscle. Three interventional protocols-cTBS150-PAS25, PAS25-cTBS150, and PAS25-alone were compared. Both cTBS150 and the TMS pulses used in PAS25 were targeted to the motor hot spot for the FDI muscle (optimal position for activation of the FDI muscle). The stimulus intensities for both stimulations (80\% active motor threshold for cTBS150 and $1 \mathrm{mV}$ for PAS25) were also determined for the FDI muscle. Importantly, the location for median nerve stimulation was unchanged.

Measurements. Measurements included resting motor threshold (RMT), MEP amplitude, recruitment curve, and intracortical circuits (Fig. 1). RMT in APB muscle was defined as the lowest TMS intensity needed to generate MEPs of $>50 \mu \mathrm{V}$ in at least 5 of 10 trials when the muscle was completely relaxed. MEP amplitude was measured with $1 \mathrm{mV}$ TMS intensity determined before each intervention. MEP recruitment curve was measured with TMS at 100, 120, 140, and 160\% RMT. The TMS intensity was adjusted at each time point if RMT changed. Four Magstim $200^{2}$ stimulators (for four TMS intensities) and a custom-made module (four-to-one connection box) were used for the measurement of recruitment curve. Ten trials for each TMS intensity (40 trials in total) were delivered in a random order. The intracortical circuits measured were short-interval intracortical inhibition (SICI), intracortical facilitation (ICF), long-interval intracortical inhibition (LICI) and short- 
latency afferent inhibition (SAI) with a pairedpulse (conditioning test) TMS paradigm ( $\mathrm{Ni}$ et al., 2011b). The test pulse intensity was set at 1 $\mathrm{mV}$ adjusted as needed at each time point. For SICI and ICF, conditioning pulse intensity was set at 80\% RMT (Kujirai et al., 1993). Interstimulus interval was $2 \mathrm{~ms}$ for SICI and $10 \mathrm{~ms}$ for ICF. For LICI, the conditioning intensity was also set at $1 \mathrm{mV}$ and interstimulus interval was $100 \mathrm{~ms}$ (Wassermann et al., 1996). For SAI, the conditioning pulse was median nerve stimulation at three times sensory threshold. The interstimulus interval was the N20 latency +3 ms. N20 latency was measured before the first experimental session by averaging 200 somatosensory evoked potentials, which was recorded with electrodes placed at $\mathrm{CP} 3$ (active) and $\mathrm{Fz}$ (reference) according to the international 10-20 system (Tokimura et al., 1996; Sailer et al., 2003). The stimulus configuration for testing of intracortical circuits consisted of six conditions. There were four paired-pulse conditions for measurements of different intracortical circuits and test stimulus alone with adjusted $1 \mathrm{mV}$. Another condition with fixed TMS intensity set at $1 \mathrm{mV}$ measured before intervention was added to investigate the changes in MEP amplitude with the different interventional protocols. Ten trials for each condition were delivered in a random order.

Data analysis and statistical analysis. MEP amplitudes were measured peak to peak. For the intracortical circuits, MEP amplitude evoked by paired-pulse stimulation was expressed as a percentage of the mean MEP amplitude of test stimulus alone. Values $<100 \%$ indicate inhibition and values $>100 \%$ indicate facilitation. Values are reported as mean $\pm \mathrm{SE}$.

A two-way repeated-measures ANOVA was used to examine the effects of interventional protocol and time on the different measurements. For MEP recruitment curve, a three-way ANOVA with an additional factor of TMS intensity was performed. Post hoc paired $t$ tests with Bonferroni's correction for multiple comparisons were used to test at which time point the measurement was different among various interventional protocols if ANOVA showed significant main effects or interactions. In addition, the peak changes in MEP amplitude after different interventional protocols (largest increase or decrease in MEP amplitude as percentage to baseline) were entered into a Pearson's correlation model to test the relationship among these interventional protocols. The threshold for significance was set at $p<0.05$. StatView 5.0.1 software (SAS Institute) was used for statistical analysis.

\section{Results}

RMT was $52.1 \pm 2.4 \%$ of stimulator output at baseline (before intervention). It did not show significant differences for the different interventional protocols or at different time points. The first control experiment showed that cTBS150 alone did not produce any significant effects in any measurements (MEP amplitude, $F_{(5,45)}=0.45, p=0.808$; SICI, $F_{(5,45)}=0.39, p=0.853$; LICI, $F_{(5,45)}=0.75, p=0.593 ; \mathrm{SAI}, F_{(5,45)}=0.25, p=0.761$; ICF, $F_{(5,45)}=0.71, p=0.617$; results for ANOVA testing the effect of time course).

\section{MEP amplitude}

Figure $2 A$ showed that PAS25-induced MEP facilitation was modulated by cTBS150 applied before or after PAS25. For the target APB muscle, ANOVA revealed significant main effects of interventional protocol $\left(F_{(2,100)}=8.06, p=0.003\right)$ and time $\left(F_{(5,100)}=2.75, p=0.028\right)$ and a significant interaction between two main factors $\left(F_{(10,100)}=2.27, p=0.019\right)$. Post hoc tests confirmed that cTBS150 preceding PAS25 increased the PAS25induced LTP-like effects at T0-T45 $(p<0.01$ for T15 and $p<$ 0.05 for other time points), while cTBS150 following PAS25 canceled or even reversed the LTP-like effects at T15-T45 $(p<0.05$ for all comparisons between PAS25-cTBS150 and PAS25 alone). Figure $2 B$ showed that the postintervention effects produced by PAS10 were also modulated by cTBS150. There was significant main effect of interventional protocol $\left(F_{(2,90)}=7.80, p=0.004\right)$ but not of time. The interaction between the two main factors was significant $\left(F_{(10,90)}=3.13, p=0.002\right)$. Post hoc tests found that cTBS150 both preceding and following PAS10 reversed the sign of LTD-like effect produced by PAS10 at T0 and T15 $(p<0.01$ for comparison between cTBS150-PAS10 and PAS10 alone at $\mathrm{T} 15, p<0.05$ for the other comparisons).

When TMS pulse used in PAS was targeted on the APB muscle, MEP in the nontarget FDI muscle increased after PAS25 and decreased after PAS10 for all three interventional protocols tested (cTBS150-PAS, PAS-cTBS150, and PAS alone; Fig. 2). ANOVA showed significant changes with time for both the PAS25induced $\left(F_{(5,100)}=3.46, p=0.009\right)$ and the PAS10-induced effects $\left(F_{(5,90)}=3.12, p=0.017\right)$. However, the main effect for the three different interventional protocols and the interaction between time and interventional protocols were not significant. This raised the possibility that the modulation of PAS25 and PAS10 by cTBS150 might be specific to the targeted muscle of 


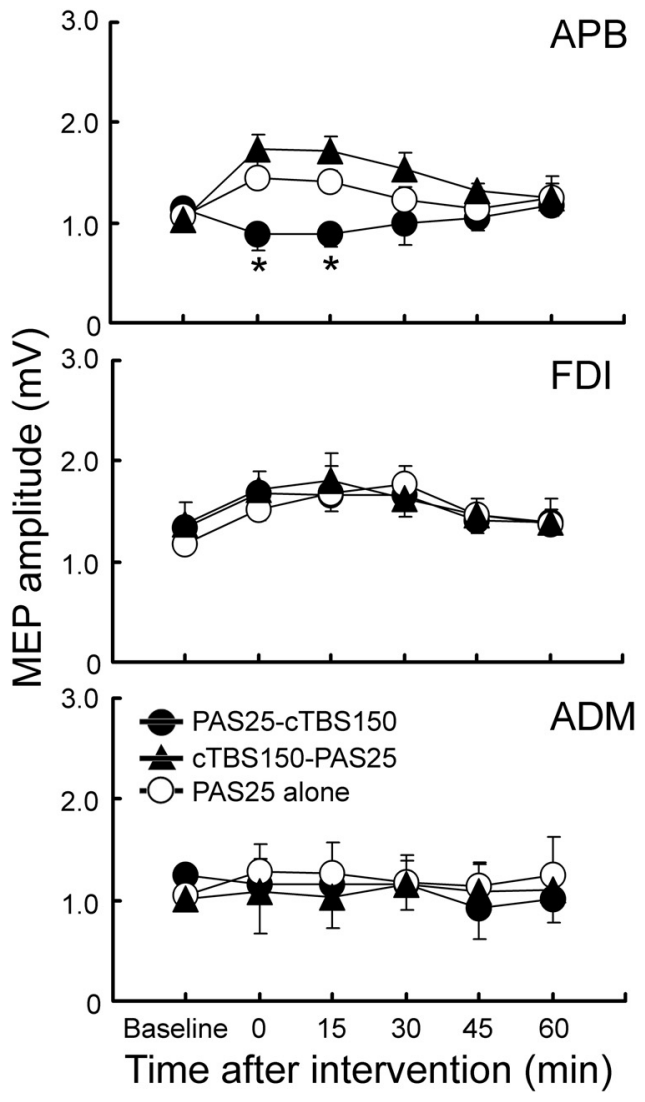

Figure 3. Effects of interventional protocols on MEP amplitude with FDI muscle as the TMS target. The abscissa indicates time and the ordinate indicates MEP amplitude. The open circles represent the interventional protocol of PAS25 alone. The filled circles represent the interventional protocol of PAS25-CTBS150. The filled triangles represent the interventional protocol of CTBS150-PAS25. Both CTBS150 and TMS used in PAS25 were targeted on the FDI muscle. MEP amplitudes in APB (top), FDI (middle), and ADM (bottom) muscles were recorded. ${ }^{*} p<0.05$, comparing PAS25-cTBS150 to PAS25 alone. CTBS150, cTBS with 150 pulses; PAS25, PAS with 25 ms interstimulus interval between median nerve stimulation and TMS.

TMS, and weak cTBS150 intensity for the nontargeted FDI muscle might not be strong enough to modulate the PAS-induced effect. To further test this possibility, we performed a second control experiment. Figure 3 showed that the facilitatory effect produced by PAS25 in the FDI muscle was not altered by the priming or following cTBS150 even though cTBS150 and PAS25 were targeted to the FDI representation. ANOVA only revealed significant effect of time $\left(F_{(5,60)}=3.61, p=0.011\right)$ but no significant effect of interventional protocol or interaction between the two main factors. On the other hand, significant main effect of interventional protocol $\left(F_{(2,60)}=6.32, p=0.013\right)$ was found in the APB muscle with a trend toward significance for the main effect of time $\left(F_{(5,60)}=2.07, p=0.098\right)$ and a significant interaction between the two factors $\left(F_{(10,60)}=3.81, p<0.001\right)$, although the FDI muscle was the TMS target for cTBS150 and TMS pulse used in PAS25. Post hoc testing showed that a following cTBS150 canceled the PAS25-induced LTP-like effect at T0 and T15 ( $p<0.05$ for both comparisons between PAS25-cTBS150 and PAS25 alone). The difference between cTBS150-PAS25 and PAS25 alone did not reach significance. This may be because TMS was targeted to the FDI muscle and the number of subjects was relatively small. MEP in the ADM muscle did not change with any interventional protocol at any time point whether the TMS target was APB or FDI muscle.
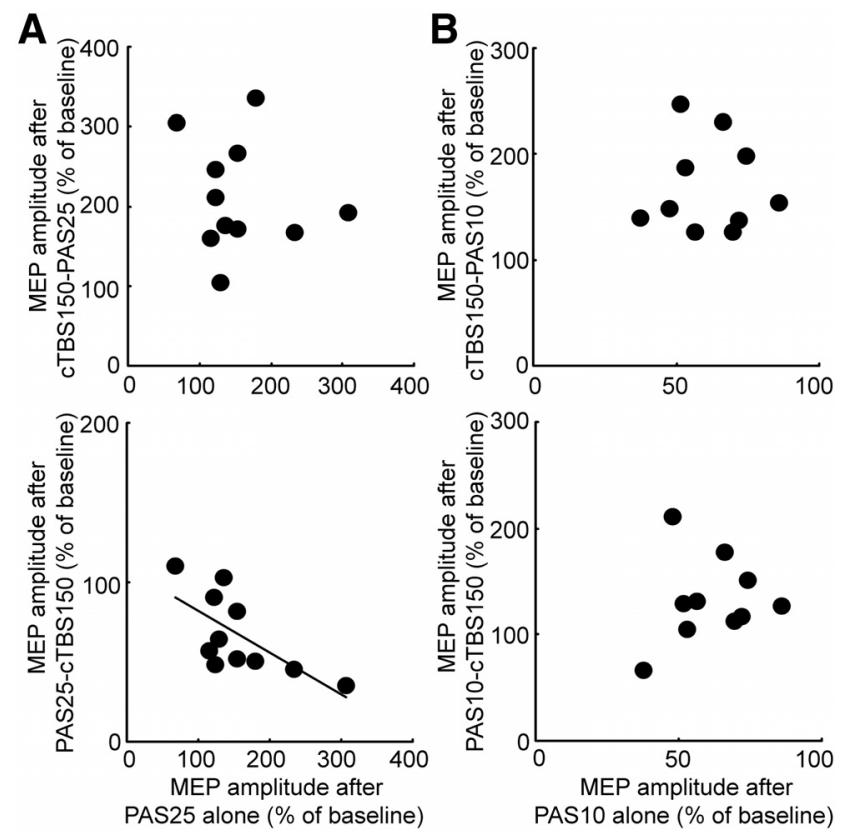

Figure 4. Relationship between MEP amplitudes after different interventional protocols in APB muscle. Modulations on PAS25- ( $\boldsymbol{A}$ ) and PAS10 ( $\boldsymbol{B}$ )-induced effects by CTBS150 were tested. The abscissas indicate the peak MEP amplitudes after the interventional protocols of PAS25 alone $(\boldsymbol{A})$ and after PAS10 alone $(\boldsymbol{B})$. The ordinates indicate the peak MEP amplitudes after the interventional protocol of CTBS150-PAS25 or CTBS150-PAS10 (top) and the peak MEP amplitudes after the interventional protocol of PAS25-CTBS150 or PAS10-cTBS150 (bottom). The values were expressed as the percentage of MEP amplitude after the interventional protocol to the MEP amplitude measured before the interventional protocol (baseline). The dark line ( $\boldsymbol{A}$, bottom) represents a regression line indicating a positive correlation between peak inhibition after PAS25-CTBS150 (lower value of MEP amplitude represents larger effect of inhibition) and peak facilitation after PAS25 alone; $p<0.05$. CTBS150, cTBS with 150 pulses; PAS25 (or PAS10), PAS with $25 \mathrm{~ms}$ (or $10 \mathrm{~ms}$ ) interstimulus interval between median nerve stimulation and TMS.

Further correlation analysis in the APB muscle (when APB muscle was the target; Fig. $4 A$ ) showed that the peak decrease in MEP amplitude after PAS25-cTBS150 significantly correlated with the peak increase in MEP amplitude after PAS25 alone $(r=$ $\left.-0.678, F_{(1,9)}=7.66, p=0.022\right)$. The peak increase in MEP amplitude after cTBS150-PAS25 did not correlate with that after PAS25 alone. On the other hand, the changes in PAS10-induced effect produced either by priming or following cTBS150 did not correlate with the effect produced by PAS10 alone (Fig. 4B).

\section{MEP recruitment curve}

Figure 5 showed that the postintervention effects in the APB muscle were different depending on the TMS intensities, with greater effects at higher stimulus intensities. Three-way ANOVA revealed significant main effects of interventional protocol $\left(F_{(2,300)}=5.26, p=0.015\right)$ and stimulus intensity $\left(F_{(3,300)}=\right.$ $63.18, p<0.001)$ but not time for PAS25 compared with that primed and followed by cTBS150. There were also significant interactions between intervention and time $\left(F_{(10,300)}=2.25, p=\right.$ $0.020)$ and between intervention and stimulus intensity $\left(F_{(6,300)}\right.$ $=3.53, p=0.005)$. The interaction between time and intensity and the three-way interaction were not significant. Post hoc tests confirmed that a preceding cTBS150 increased the PAS25induced MEP facilitation for 140\% RMT TMS intensity at T15 $(p<0.05)$, T30 $(p<0.01)$, and for $160 \%$ RMT at T0, T15 $(p<$ $0.05)$, and T30 $(p<0.01)$. A following cTBS150 canceled this MEP facilitation for $120 \%$ RMT at T15 $(p<0.05)$ and for 140 
and $160 \%$ RMT at T0 $(p<0.05)$ and T15 $(p<0.01)$, respectively. The modulation on PAS10-induced effects with cTBS150 was also dependent on TMS intensity $\left(F_{(3,270)}=37.09, p<0.001\right)$. ANOVA revealed trend toward significance for main effect of interventional protocol $\left(F_{(2,270)}\right.$ $=2.74, p=0.092)$. The main effect of time was not significant. There were significant interactions between intervention and time $\left(F_{(10,270)}=2.51, p=0.010\right)$, between intervention and stimulus inten$\operatorname{sity}\left(F_{(6,270)}=2.49, p=0.033\right)$, and a significant three-way interaction $\left(F_{(30,270)}=\right.$ $1.89, p=0.005)$. The interaction between time and stimulus intensity was not significant. Post hoc tests found that the priming cTBS150 reversed the sign of PAS10 for $140 \%$ RMT and 160\% RMT TMS intensity at T0 $(p<0.01$ for both comparisons) and T15 ( $p<0.05$ for both comparisons). In addition, cTBS150 following PAS10 reversed the LTD-like effect for $140 \%$ RMT TMS intensity at T0 and for $160 \%$ RMT TMS intensity at T0 and T15 ( $p<0.05$ for all comparisons).

\section{Intracortical circuits}

In the target APB muscle, PAS25 alone did not change SICI and LICI. However, both intracortical inhibitory circuits were reduced by cTBS150-PAS25 and were enhanced by PAS25-cTBS150 protocol (Fig. $6 A)$. ANOVA revealed significant main effect of interventional protocol $\left(F_{(2,100)}\right.$ $=3.63, p=0.045)$ and interaction between intervention and time $\left(F_{(10,100)}=\right.$ 2.24, $p=0.021$ ) for SICI. The main effect of time was not significant. Post hoc test confirmed that SICI was decreased by cTBS150-PAS25 compared with PAS25 alone at T0 $(p<0.05)$ and T15 $(p<0.01)$, while SICI after PAS25-cTBS150 was increased compared with SICI after PAS25 alone at T0 and T15 ( $p<0.05$ for both comparisons). For LICI, the main effect of interventional protocol showed a trend toward significance $\left(F_{(2,100)}=2.77, p=0.087\right)$ with a significant interaction between intervention and time $\left(F_{(10,100)}=2.14, p=\right.$ 0.028). The main effect of time was not significant. Post hoc tests showed that LICI after cTBS150-PAS25 was weaker than that after PAS25 alone at T0 and T15 $(p<0.05$ for both comparisons), while LICI after PAS25-cTBS150 was stronger than that after PAS25 alone at T0 $(p<0.05)$ and T15 $(p<0.01)$. Figure $6 B$ showed that SICI was reduced by the cTBS150-PAS10 protocol but was not changed by PAS10-cTBS150 and PAS10 alone. ANOVA revealed a significant main effect of interventional protocol $\left(F_{(2,90)}=4.31, p=0.030\right)$ but not time. The interaction between two main factors was also significant $\left(F_{(10,90)}=2.46, p=\right.$ 0.012). Post hoc test confirmed that SICI was decreased by cTBS150-PAS10 compared with PAS10 alone at T0 and T15 $(p<$ 0.05 for both comparisons). Modulation of PAS10 with either priming or following cTBS150 did not change LICI. SAI and ICF did not change with any interventional protocol, as no significant main effects or interactions were found for these two circuits.

\section{Discussion}

We found that a priming cTBS150 with heterosynaptic inputs enhanced the LTP-like effects of PAS25 and reversed the LTDlike effects of PAS10. cTBS150 following PAS25 canceled the effect of PAS25 while that following PAS10 produced LTP-like effects.

\section{Heterosynaptic modulation of plasticity}

MEP amplitude significantly increased after PAS25 and decreased after PAS10 in our study, confirming the spike timingdependent plasticity reported in previous studies (Stefan et al., 2000, 2002; Wolters et al., 2003). The Bienenstock-Cooper-Munro theory proposes that metaplasticity modulates plasticity induction. It predicts that reduction in the postsynaptic activity decreases the threshold for LTP induction and facilitates the effects of a subsequently delivered LTP protocol (Bienenstock et al., 
A
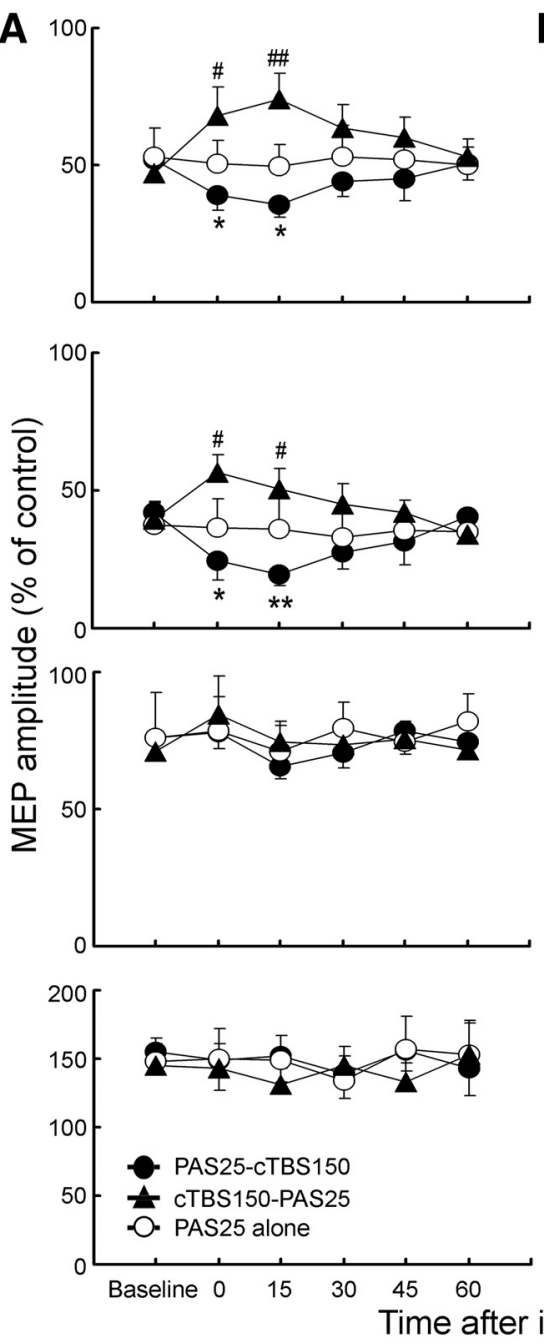

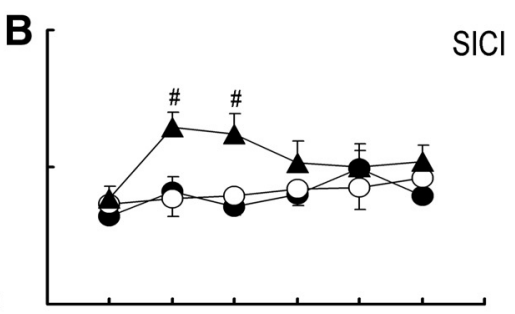

(n)
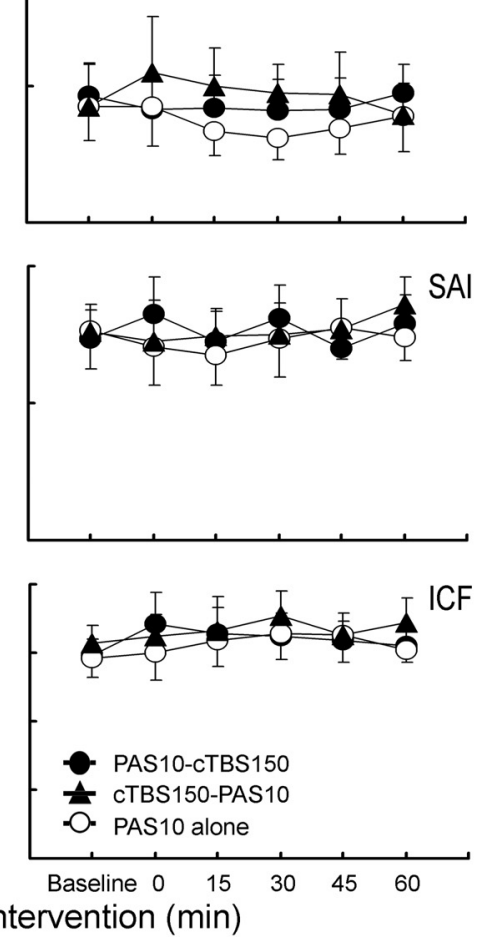

Figure 6. Effects of interventional protocols on intracortical circuits in APB muscle. Modulations on PAS25- $(\boldsymbol{A})$ and PAS10 $(\boldsymbol{B})$-induced effects by CTBS150 were tested. The effects of different interventional protocols on SICI, LICI, SAI, and ICF were investigated. The abscissa indicates time and the ordinate indicates conditioned MEP amplitudes expressed as the percentage of the MEP amplitudes induced by test stimulus alone. The open circles represent the interventional protocols of PAS25 alone $(A)$ and PAS10 alone $(\boldsymbol{B})$. The filled circles represent the interventional protocols of PAS25-CTBS150 (A) and PAS10-CTBS150 (B). The filled triangles represent the interventional protocols of CTBS150-PAS25 (A) and CTBS150-PAS10 (B). ${ }^{*} p<0.05$, ${ }^{* *} p<0.01$, comparing PAS25-cTBS150 to PAS25 alone. \#p $<0.05$, \#\#p $<0.01$, comparing cTBS150-PAS25 to PAS25 alone or comparing cTBS150-PAS10 to PAS10 alone. CTBS150, cTBS with 150 pulses; PAS25 (or PAS10), PAS with $25 \mathrm{~ms}$ (or $10 \mathrm{~ms}$ ) interstimulus interval between median nerve stimulation and TMS.

1982). In support of this theory, a previous report demonstrated that homosynaptic priming inputs with quadripulse TMS delivered at long interstimulus intervals, which produced LTD-like effects, facilitated the LTP-like effects produced by a second quadripulse TMS delivered in a shorter interstimulus interval (Hamada et al., 2008). In addition, subthreshold $1 \mathrm{~Hz}$ repetitive TMS, which had little effect by itself, when primed by cathodal transcranial direct current stimulation, which produced LTDlike effects, led to LTP-like effects (Siebner et al., 2004). This raised the possibility that heterosynaptic inputs might be effective in modulating the threshold for LTP induction as different cortical neurons were likely activated by TMS and direct current stimulation (Hallett, 2007). We found that a priming cTBS150 increased the effect of PAS25 and reversed the effect of PAS10 (Fig. 2). Since the stronger TMS used in PAS would have activated neuronal elements not activated by the much weaker TMS used in cTBS150 and sensory input was involved in PAS but not in
cTBS150, our study is the first to conclusively demonstrate the modulation of cortical plasticity (metaplasticity) by a priming heterosynaptic input. MEP recruitment curve showed that different MEP amplitude with priming cTBS150 compared with PAS alone was most pronounced at middle to high TMS intensities (Fig. 5, 1.4 and 1.6 RMT), suggesting that the cortical neurons with high firing threshold might be involved in producing the additional facilitation when PAS was primed by cTBS150. The involvement of additional cortical neurons after cTBS150-PAS protocol compared with PAS alone may also explain why the peak increase in MEP facilitation with the cTBS150-PAS protocol did not correlate with the MEP amplitude with PAS alone (Fig. 4). It should be noted that monophasic pulses, which induced posterioranterior current, were used for PAS while biphasic pulses, which induced both posterior-anterior and anterior-posterior currents, were used for cTBS150. Since different neuronal populations are activated by different current directions ( $\mathrm{Ni}$ et al., 2011a), modulation of PAS by cTBS150 may involve complex interactions between different neuronal populations. In this regard, recent studies reported that PAS protocols pairing posterior parietal cortical stimulation with motor cortical stimulation in different current directions could produce cortical plasticity either in a Hebbian's or an antiHebbian's manner (Koch et al., 2013; Veniero et al., 2013). cTBS with $<600$ pulses might produce LTP-like effect (Gentner et al., 2008), although control Experiment 1 did not show any measurable effect of cTBS150 alone. This may explain the LTP-like effect after the PAS10-cTBS150 protocol, because a strong LTD-like protocol (PAS10) reduces the threshold for LTP induction when it primes cTBS150.

A previous study reported that LTP-like effect produced by intermittent TBS was depotentiated by a following homosynaptic inputs produced by cTBS150 (Huang et al., 2010). In vivo and in vitro investigations with application of two synaptic inputs suggested that depotentiation with heterosynaptic inputs occurs at the cellular level (Fujii et al., 1991; Huang et al., 1999; Stäubli and Scafidi, 1999). Our results now showed that at the systems level in human LTP was reversible with heterosynaptic inputs. The findings supporting this notion included that PAS25 alone produced LTP-like effects and cTBS150 alone did not produce measurable changes, but cTBS150 delivered immediately after PAS25 reversed the effects of PAS25 (Fig. 2A). Moreover, the degree of reversal in MEP facilitation after PAS25-cTBS150 correlated with the degree of MEP facilitation after PAS25 alone (Fig. 4A). Depotentiation acts as a rapid brake on synaptic plasticity and is important in preventing spurious information from being stored in memory process when neural firing incidentally increases (Zhou 
and Poo, 2004). Our results suggested that large LTP caused by the incident increase in synaptic signal is particularly suppressed by depotentiation.

All three interventional protocols produced similar effects in the FDI muscle (Fig. 2), indicating that cTBS150 did not induce metaplasticity or depotentiation in the cortical representation of adjacent muscle. This was confirmed even when the FDI muscle was the target for TMS tested in the second control experiment. The results strongly suggest that heterosynaptic modulation (metaplasticity and depotentiation) represents a form of plasticity with high degree of topographic specificity, requiring median nerve stimulation and TMS to be applied to the same muscle.

\section{Bidirectional versus unidirectional modulation of plasticity}

While priming and following cTBS150 modulated the LTP-like effects of PAS25 in a bidirectional manner, both priming and following cTBS150 changed cortical excitability in the same direction for PAS10 and turned the LTD-like effect into an LTP-like effect. These results suggest that LTD is not a reverse of LTP and modulations of LTD and LTP by heterosynaptic inputs are different. This may be similar to the finding that voluntary muscle contraction produced similar directional changes for intermittent and continuous TBS. Muscle contraction during TBS blocked the LTP-like effect of intermittent TBS and the LTD-like effect of continuous TBS, while muscle contraction immediately after TBS produced LTP-like effect for both intermittent and continuous TBS (Huang et al., 2008).

\section{Changes in GABAergic circuits}

SICI is likely mediated by $\mathrm{GABA}_{\mathrm{A}}$ receptors (Ziemann et al., 1996; Di Lazzaro et al., 2005a) and LICI is likely mediated by GABA $_{B}$ receptors (Valls-Solé et al., 1992; Wassermann et al., 1996; McDonnell et al., 2006). Our results that SICI and LICI did not change after PAS alone are consistent with previous studies (Stefan et al., 2002; Rosenkranz and Rothwell, 2006). Both SICI and LICI decreased after cTBS150-PAS25 and increased after PAS25cTBS150 protocol. These results suggest that modulations of LTP induction with metaplasticity and depotentiation by heterosynaptic inputs are associated with changes in GABAergic circuits. Similarly, LTP induction with $0.1 \mathrm{~Hz}$ repetitive TMS primed by ischemic nerve block is accompanied by reduction in SICI (Ziemann et al., 1998) while engagement of SICI during PAS25 blocks LTP induction (Elahi et al., 2012). Cellular studies in the piriform and motor cortices in rats found that blockade of GABA receptors led to facilitation of LTP induction (Kanter and Haberly, 1993; Hess et al., 1996). The present study provided further evidence that GABAergic circuits were involved in the modulation of LTD. Particularly, decreased SICI after cTBS150-PAS10 may explain why it produced LTP-like rather than LTD-like effects. The lower degree of LTP-like effect after PAS10-cTBS150 may be related to the unchanged SICI. These findings are consistent with GABA being an important gate keeper of cortical plasticity (Sanes and Donoghue, 2000; Abraham, 2008).

SAI is mediated by cholinergic and GABAergic pathways activated by afferent inputs (Di Lazzaro et al., 2000, 2005b; Tokimura et al., 2000; Sailer et al., 2003 ). Although cortical excitatory glutamatergic interneurons may be involved in ICF (Ziemann, 2004), its mechanism remains unclear as epidural recordings show that ICF is not associated with changes in descending corticospinal volleys (Di Lazzaro et al., 2006). We did not find changes in SAI and ICF with any of our interventional protocol, suggesting that they do not contribute to the processes of metaplasticity or depotentiation.

\section{Homeostatic plasticity in motor cortical neurons}

The Hebbian theory that changes in synaptic efficacy of the postsynaptic neuron arise from the repeated stimulation of the presynaptic neuron in a certain time window (Hebb, 1949) explains the spike timing-dependent plasticity produced by PAS (Stefan et al., 2000, 2002). However, the persistence of LTP could lead to a saturation of all modifiable synapses in a potentiated state, making it impossible to store additional new information (Turrigiano, 2008). Homeostatic plasticity, which maintains synaptic weight and neuronal activity in a dynamic physiological range, keeps cortical plasticity in a flexible range. Heterosynaptic modulation revealed in the present study represents a powerful form of homeostatic cortical plasticity with cross-compartment modification for spike timing-dependent plasticity. However, modulation of cortical plasticity by heterosynaptic inputs is a complex process. It was reported that cortical plasticity was modulated nonhomeostatically when PAS was primed by transcranial direct current stimulation while there was homeostatic interaction when the two protocols were applied simultaneously (Nitsche et al., 2007). Similarly, nonhomeostatic and homeostatic interactions occurred when PAS primed motor practice without and with a time delay (Jung and Ziemann, 2009). Although the exact mechanism is not known, the inhibitory cTBS150 on one group of synapses may have bidirectional effects at other groups of synapses. In conclusion, our study identified at the systems level that heterosynaptic inputs modulate the motor cortical neurons, which follows the principles of metaplasticity and depotentiation and are accompanied by the changes in GABAergic interneuron excitability, and such homeostatic modulation is determined by the order of the interventions used.

\section{References}

Abraham WC (2008) Metaplasticity: tuning synapses and networks for plasticity. Nat Rev Neurosci 9:387-399. CrossRef Medline

Bienenstock EL, Cooper LN, Munro PW (1982) Theory for the development of neuron selectivity: orientation specificity and binocular interaction in visual cortex. J Neurosci 2:32-48. Medline

Chistiakova M, Volgushev M (2009) Heterosynaptic plasticity in the neocortex. Exp Brain Res 199:377-390. CrossRef Medline

Di Lazzaro V, Oliviero A, Profice P, Pennisi MA, Di Giovanni S, Zito G, Tonali P, Rothwell JC (2000) Muscarinic receptor blockade has differential effects on the excitability of intracortical circuits in the human motor cortex. Exp Brain Res 135:455-461. CrossRef Medline

Di Lazzaro V, Oliviero A, Saturno E, Pilato F, Insola A, Mazzone P, Profice P, Tonali P, Rothwell JC (2001) The effect on corticospinal volleys of reversing the direction of current induced in the motor cortex by transcranial magnetic stimulation. Exp Brain Res 138:268-273. CrossRef Medline

Di Lazzaro V, Oliviero A, Saturno E, Dileone M, Pilato F, Nardone R, Ranieri F, Musumeci G, Fiorilla T, Tonali P (2005a) Effects of lorazepam on short latency afferent inhibition and short latency intracortical inhibition in humans. J Physiol 564:661-668. CrossRef Medline

Di Lazzaro V, Pilato F, Dileone M, Tonali PA, Ziemann U (2005b) Dissociated effects of diazepam and lorazepam on short-latency afferent inhibition. J Physiol 569:315-323. CrossRef Medline

Di Lazzaro V, Pilato F, Oliviero A, Dileone M, Saturno E, Mazzone P, Insola A, Profice P, Ranieri F, Capone F, Tonali PA, Rothwell JC (2006) Origin of facilitation of motor-evoked potentials after paired magnetic stimulation: direct recording of epidural activity in conscious humans. J Neurophysiol 96:1765-1771. CrossRef Medline

Elahi B, Gunraj C, Chen R (2012) Short-interval intracortical inhibition blocks long-term potentiation induced by paired associative stimulation. J Neurophysiol 107:1935-1941. CrossRef Medline

Fujii S, Saito K, Miyakawa H, Ito K, Kato H (1991) Reversal of long-term potentiation (depotentiation) induced by tetanus stimulation of the input to CA1 neurons of guinea pig hippocampal slices. Brain Res 555:112-122. CrossRef Medline

Gentner R, Wankerl K, Reinsberger C, Zeller D, Classen J (2008) Depression of human corticospinal excitability induced by magnetic theta-burst 
stimulation: evidence of rapid polarity-reversing metaplasticity. Cereb Cortex 18:2046-2053. CrossRef Medline

Hallett M (2007) Transcranial magnetic stimulation: a primer. Neuron 55: 187-199. CrossRef Medline

Hamada M, Terao Y, Hanajima R, Shirota Y, Nakatani-Enomoto S, Furubayashi T, Matsumoto H, Ugawa Y (2008) Bidirectional long-term motor cortical plasticity and metaplasticity induced by quadripulse transcranial magnetic stimulation. J Physiol 586:3927-3947. CrossRef Medline

Hebb D (1949) Organization of behavior. New York: Wiley.

Hess G, Aizenman CD, Donoghue JP (1996) Conditions for the induction of long-term potentiation in layer II/III horizontal connections of the rat motor cortex. J Neurophysiol 75:1765-1778. Medline

Huang CC, Liang YC, Hsu KS (1999) A role for extracellular adenosine in time-dependent reversal of long-term potentiation by low-frequency stimulation at hippocampal CA1 synapses. J Neurosci 19:9728-9738. Medline

Huang YZ, Edwards MJ, Rounis E, Bhatia KP, Rothwell JC (2005) Theta burst stimulation of the human motor cortex. Neuron 45:201-206. CrossRef Medline

Huang YZ, Rothwell JC, Edwards MJ, Chen RS (2008) Effect of physiological activity on an NMDA-dependent form of cortical plasticity in human. Cereb Cortex 18:563-570. CrossRef Medline

Huang YZ, Rothwell JC, Lu CS, Chuang WL, Lin WY, Chen RS (2010) Reversal of plasticity-like effects in the human motor cortex. J Physiol 588: 3683-3693. CrossRef Medline

Huang YZ, Rothwell JC, Lu CS, Chuang WL, Chen RS (2011) Abnormal bidirectional plasticity-like effects in Parkinson's disease. Brain 134:23122320. CrossRef Medline

Jung P, Ziemann U (2009) Homeostatic and nonhomeostatic modulation of learning in human motor cortex. J Neurosci 29:5597-5604. CrossRef Medline

Kaneko K, Kawai S, Fuchigami Y, Morita H, Ofuji A (1996) The effect of current direction induced by transcranial magnetic stimulation on the corticospinal excitability in human brain. Electroencephalogr Clin Neurophysiol 101:478-482. CrossRef Medline

Kanter ED, Haberly LB (1993) Associative long-term potentiation in piriform cortex slices requires GABAA blockade. J Neurosci 13:2477-2482. Medline

Koch G, Ponzo V, Di Lorenzo F, Caltagirone C, Veniero D (2013) Hebbian and anti-Hebbian spike-timing-dependent plasticity of human corticocortical connections. J Neurosci 33:9725-9733. CrossRef Medline

Kujirai T, Caramia MD, Rothwell JC, Day BL, Thompson PD, Ferbert A, Wroe S, Asselman P, Marsden CD (1993) Corticocortical inhibition in human motor cortex. J Physiol 471:501-519. Medline

Kulla A, Manahan-Vaughan D (2000) Depotentiation in the dentate gyrus of freely moving rats is modulated by D1/D5 dopamine receptors. Cereb Cortex 10:614-620. CrossRef Medline

Malenka RC, Bear MF (2004) LTP and LTD: an embarrassment of riches. Neuron 44:5-21. CrossRef Medline

McDonnell MN, Orekhov Y, Ziemann U (2006) The role of GABA(B) receptors in intracortical inhibition in the human motor cortex. Exp Brain Res 173:86-93. CrossRef Medline

Ni Z, Charab S, Gunraj C, Nelson AJ, Udupa K, Yeh IJ, Chen R (2011a) Transcranial magnetic stimulation in different current directions activates separate cortical circuits. J Neurophysiol 105:749-756. CrossRef Medline

Ni Z, Müller-Dahlhaus F, Chen R, Ziemann U (2011b) Triple-pulse TMS to study interactions between neural circuits in human cortex. Brain Stimul 4:281-293. CrossRef Medline

Nitsche MA, Roth A, Kuo MF, Fischer AK, Liebetanz D, Lang N, Tergau F, Paulus W (2007) Timing-dependent modulation of associative plastic- ity by general network excitability in the human motor cortex. J Neurosci 27:3807-3812. CrossRef Medline

Oldfield RC (1971) The assessment and analysis of handedness: The Edinburgh inventory. Neuropsychologia 9:97-113. CrossRef Medline

Rosenkranz K, Rothwell JC (2006) Differences between the effects of three plasticity inducing protocols on the organization of the human motor cortex. Eur J Neurosci 23:822-829. CrossRef Medline

Sailer A, Molnar GF, Paradiso G, Gunraj CA, Lang AE, Chen R (2003) Short and long latency afferent inhibition in Parkinson's disease. Brain 126: 1883-1894. CrossRef Medline

Sanes JN, Donoghue JP (2000) Plasticity and primary motor cortex. Annu Rev Neurosci 23:393-415. CrossRef Medline

Siebner HR, Lang N, Rizzo V, Nitsche MA, Paulus W, Lemon RN, Rothwell JC (2004) Preconditioning of low-frequency repetitive transcranial magnetic stimulation with transcranial direct current stimulation: evidence for homeostatic plasticity in the human motor cortex. J Neurosci 24:3379-3385. CrossRef Medline

Stäubli U, Scafidi J (1999) Time-dependent reversal of long-term potentiation in area CA1 of the freely moving rat induced by theta pulse stimulation. J Neurosci 19:8712-8719. Medline

Stefan K, Kunesch E, Cohen LG, Benecke R, Classen J (2000) Induction of plasticity in the human motor cortex by paired associative stimulation. Brain 123:572-584. CrossRef Medline

Stefan K, Kunesch E, Benecke R, Cohen LG, Classen J (2002) Mechanisms of enhancement of human motor cortex excitability induced by interventional paired associative stimulation. J Physiol 543:699-708. CrossRef Medline

Tokimura H, Ridding MC, Tokimura Y, Amassian VE, Rothwell JC (1996) Short latency facilitation between pairs of threshold magnetic stimuli applied to human motor cortex. Electroencephalogr Clin Neurophysiol 101:263-272 CrossRef Medline

Tokimura H, Di Lazzaro V, Tokimura Y, Oliviero A, Profice P, Insola A, Mazzone P, Tonali P, Rothwell JC (2000) Short latency inhibition of human hand motor cortex by somatosensory input from the hand. J Physiol 523:503-513. CrossRef Medline

Turrigiano GG (2008) The self-tuning neuron: synaptic scaling of excitatory synapses. Cell 135:422-435. CrossRef Medline

Valls-Solé J, Pascual-Leone A, Wassermann EM, Hallett M (1992) Human motor evoked responses to paired transcranial magnetic stimuli. Electroencephalogr Clin Neurophysiol 85:355-364. CrossRef Medline

Veniero D, Ponzo V, Koch G (2013) Paired associative stimulation enforces the communication between interconnected areas. J Neurosci 33:1377313783. CrossRef Medline

Wassermann EM, Samii A, Mercuri B, Ikoma K, Oddo D, Grill SE, Hallett M (1996) Responses to paired transcranial magnetic stimuli in resting, active, and recently activated muscle. Exp Brain Res 109:158-163. Medline

Wolters A, Sandbrink F, Schlottmann A, Kunesch E, Stefan K, Cohen LG, Benecke R, Classen J (2003) A temporally asymmetric Hebbian rule governing plasticity in the human motor cortex. J Neurophysiol 89:23392345. CrossRef Medline

Zhou Q, Poo MM (2004) Reversal and consolidation of activity-induced synaptic modifications. Trends Neurosci 27:378-383. CrossRef Medline

Ziemann U (2004) TMS and drugs. Clin Neurophysiol 115:1717-1729. CrossRef Medline

Ziemann U, Lönnecker S, Steinhoff BJ, Paulus W (1996) The effect of lorazepam on the motor cortical excitability in man. Exp Brain Res 109:127135. Medline

Ziemann U, Corwell B, Cohen LG (1998) Modulation of plasticity in human motor cortex after forearm ischemic nerve block. J Neurosci 18: 1115-1123. Medline 